\title{
Editorial
}

\section{Infections in Long-Term-Care Facilities: Screen or Clean?}

\author{
Chesley L. Richards, Jr., MD, MPH
}

For the second time in a year, Infection Control and Hospital Epidemiology has chosen to devote several articles to infection control issues in long-term-care facilities (LTCFs), either directly or indirectly. ${ }^{1}$ Why? In part, it's the science. Maybe, in part, it's the recognition that a substantial amount of healthcare is and will increasingly be provided in the LTCFs. In the United States, 1.6 million adults, mostly frail elderly, live in LTCFs. The most common LTCFs are nursing homes. In nursing homes, "handson" care usually is provided by a nursing assistant. Nursing assistants may have several residents to feed, bathe, clean, and dress. Nursing assistants may be the most dedicated healthcare workers in a given nursing facility, but overall usually are the least trained, lowest paid workers with the highest job turnover rates. The financial resources to provide care in nursing homes for the elderly population come primarily from Medicaid and personal funds, and enormous pressures are building to limit cost growth in this segment of long-term care. These pressures are, unfortunately, translating into suboptimal staffing and minimal resources for infection control. Simply maintaining current financial resources for LTCFs is challenging and increased resources are unlikely. So, infection control strategies in LTCFs must be relatively simple, inexpensive, practical, and focused on the most important problems.

In this issue of Infection Control and Hospital Epidemiology, five articles ${ }^{2-6}$ deal directly or indirectly with issues in LTCF residents. The topics covered include methicillin-resistant Staphylococcus aureus (MRSA) and vancomycin-resistant enterococci (VRE), norovirus, and bloodstream infections. Superficially, these articles may appear to be on disparate topics. However, when taken as a whole, they push healthcare epidemiologists and infection control professionals to consider one of the dilemmas facing those of us who practice in a long-term-care environment. With limited resources, should we focus on surveillance and screening activities or on infection control interventions? Simply stated, do we use our limited resources to screen or clean?

Three of the articles explore aspects of the epidemiology or management of MRSA or VRE. In a survey of 331 Iowa nursing homes, Kreman et al. report that the majority of facilities reported accepting individuals colonized or infected with MRSA or VRE. ${ }^{2}$ Furthermore, the vast majority of nursing homes reported using standard and contact precautions, allowing residents to socialize if secretions were contained, and requiring handwashing and glove use. Although the majority reported using contact precautions, only half reported routinely placing residents in private rooms or cohorting. Many facilities reported making the decision on room placement based on patient characteristics. Almost $90 \%$ of the facilities reported performing cultures to determine whether contact precautions can be discontinued. Are cultures really necessary if contact precautions are only partially implemented? Does the natural history of VRE and MRSA really lend itself to clearance once colonization has occurred? If not, are surveillance cultures a good use of sparse resources? This study highlights the potential disconnect that exists regarding an approach to managing patients colonized or infected with MRSA or VRE.

Simor et al. report the clinical and epidemiologic characteristics of inpatients who were colonized with MRSA

Dr. Richards is from the Atlanta/Birmingham VA Geriatrics Research Educational Clinical Center (GRECC) and Atlanta VA Health Services Research and Development (HSRD), Atlanta, Georgia. He is also from the Division of Geriatric Medicine and Gerontology, Emory University School of Medicine, Atlanta, Georgia.

Address reprint requests to Chesley Richards, MD, MPH, Atlanta VA Medical Center, GRECC (11B) 1670 Clairmont Road, Atlanta, GA 30033. Chesley.Richards@va.gov 
in 37 Canadian hospitals. ${ }^{3}$ Approximately $70 \%$ of these patients acquired MRSA in the hospital. Elderly adults were more likely to be colonized and younger adults were more likely to have infection. Most of the MRSA in elderly adults was found through screening, and most of these patients had MRSA isolated from urine or perineum. As a group, do these elderly individuals really cause substantial MRSA transmission in the hospital or in the nursing home they may go to following hospitalization?

Wendt et al. report that in 47 German nursing homes, $12 \%$ to $54 \%$ of the residents were colonized with MRSA. ${ }^{4}$ The authors assessed transmission in the nursing homes by looking for pulsed-field gel electrophoresis patterns shared by two or more residents. They calculated transmission rates of $0 \%$ to $70 \%$ within individual nursing homes and found that multiple-patient rooms (3 or more patients) and nursing home stays of 6 months or more were associated with higher risk of transmission. Without specific data on functional status, it is difficult to know whether these individuals were mobile, either independently or with assistance. However, resident to healthcare worker to resident transmission was probably the most likely mechanism in this environment.

Two additional articles deal with norovirus and bloodstream infections. In an outbreak investigation of norovirus in a 250-bed veterans nursing home, Wu et al. report that $52 \%$ of the residents and $46 \%$ of the staff developed gastroenteritis. ${ }^{5}$ Extensive environmental contamination with norovirus was found and this may have perpetuated the outbreak. They draw attention to the specific issue that environmental cleaning in nursing homes should include agents active against norovirus. This is important. What also is important from this study is that in this nursing home, resident rooms and common areas (eg, dining areas and day rooms) were regularly cleaned. This is not a given in many nursing homes. As nursing homes aspire to be more like "homes" and less like "nursing homes," infection control professionals must maintain vigilance that at least they are clean "homes."

Finally, Mylotte reviews seven published studies from 1980 to 2003 on nursing home-associated bloodstream infections. ${ }^{6}$ That's right, seven studies in 23 years with a grand total of 503 patients. In these studies, nursing home bloodstream infections were infrequent (incidence, 0.3 episode per 1,000 resident-days) and the yield from blood cultures was low, in terms of both positive culture and the potential for impact on resident outcomes. Mylotte's recommendation is that blood cultures not be done routinely in most nursing homes, a recommendation that is consistent with expert-driven published guidelines. So, in other words, don't "screen" for bloodstream infections in nursing homes. If bloodstream infection is truly suspected, nursing home residents should be hospitalized or there should be a good reason against hospitalization (eg, advance directive).

So what should we make of these articles? They represent good work that is needed to expand our infection control and epidemiologic knowledge in a healthcare sector woefully in need of good science. They reinforce the notion that there is much we do not know and the recognition that there is a broad agenda for research on infections in LTCF residents.? And, I believe, they suggest that interventions such as good environmental cleaning, excellent hand hygiene, and practical translation of infection control guidelines are likely to have more impact than extensive screening or surveillance programs more appropriate for acute care settings. These interventions may be simple in concept, but they are not easily implemented or sustained. We, as an infection control community, need to understand how to do this better. LTCFs need our help with infection control, but our help should be practical and in keeping with the resources and realities of LTCFs.

\section{REFERENCES}

1. Bradley SF. Double, double, toil and trouble: infections still spreading in long-term-care facilities. Infect Control Hosp Epidemiol 2005;26:227230 .

2. Kreman T, Hu J, Pottinger J, Herwaldt LA. Survey of long-term-care facilities in Iowa for policies and practices regarding residents with methicillin-resistant Staphylococcus aureus or vancomycin-resistant enterococci. Infect Control Hosp Epidemiol 2005;26:811-815.

3. Simor AE, Ofner-Agostini M, Paton S, et al. Clinical and epidemiologic features of methicillin-resistant Staphylococcus aureus in elderly hospitalized patients. Infect Control Hosp Epidemiol 2005;26:838-841.

4. Wendt C, Svoboda D, Schmidt C, Bock-Hensley O, von Baum H. Characteristics that promote transmission of Staphylococcus aureus in German nursing homes. Infect Control Hosp Epidemiol 2005;26:816-821.

5. Wu HM, Fornek M, Schwab KJ, et al. A norovirus outbreak at a longterm-care facility: the role of environmental surface contamination. Infect Control Hosp Epidemiol 2005;26:802-810.

6. Mylotte JM. Nursing home-acquired bloodstream infection. Infect Control Hosp Epidemiol 2005;26:833-837.

7. Richards C. Infections in residents of long-term care facilities: an agenda for research. Report of an Expert Panel. J Am Geriatr Soc 2002; 50:570-576. 